Chapter 4

\title{
Control of Quantum Particle Dynamics by Impulses of Magnetic Field
}

\author{
Valeriy Efimovich Arkhincheev \\ Additional information is available at the end of the chapter \\ http://dx.doi.org/10.5772/63697
}

\begin{abstract}
In the present chapter, the possibility of spin states control in quantum dots as bases of creation of logic elements on the basis of quantum semi-conductor systems is discussed. In particular, the multi-particle approach to the description of spin system and control of coherent spin conditions is developed. It is shown that at action by an impulse of a magnetic field two types of spin conditions are possible, depending on initial data: conditionally steady and absolutely steady. The second type of conditions is important for information storage.
\end{abstract}

Keywords: spin states, control, information, stability, magnetic field, quantum dot, quantum dynamics

\section{Introduction}

Now, a lot of works on search of materials for development of qubits as bases of element base for quantum calculations has conducted. It is conducted in several directions, but the most perspective, in our opinion, possibility of creation of qubits (elements of quantum calculations) on the basis of low-dimensional quantum semiconductor systems with quantum dots is represented [1]. With search of perspective materials, the important problem is development both the new and other adapted under concrete quantum nanostructures algorithms and schemes of the quantum calculations allowing to realize logical schemes and elements. Since 1994, when Shor [2], Shor and Preskil [3], and Shor and Smolin [4] offered effective algorithm of polynomial (power) type for the solution of a problem of factorization of large numbers on quantum computers, the intensive development of algorithms of quantum calculations began. Essential feature of quantum computers is possibility of implementation of Fourier transfor- 
mation for polynomial (power) number of operations depending on the task size. In that, there is the fundamental difference of quantum calculations from classical ones, where the exponential number of operations is necessary for using of Fourier's transformations.

At the present moment, three classes of quantum algorithms are known [5-8]. The first class of algorithms is intended for the solution of tasks like factorization of numbers, the second class of algorithms is developed for modeling of the quantum phenomena [9], and the third class of algorithms is intended for search of object in an unstructured random database [10].

In this first part of the chapter, the possibility of control of spin states in quantum dots as bases of creation of logical elements on the basis of quantum semiconductor systems has discussed. In particular, multi-particle approach to the description of spin system and control of coherent spin states has developed. It has shown that at action by an impulse of a magnetic field and depending on initial data two types of spin states are possible: non-steady and absolutely steady. The second type of states is important for storage of the information.

\section{Physical basics for development of control of spin system}

\subsection{Matrix representation of spin operators in multiparticle approach}

To solve the control problem of many-particle spin system, we need to develop the manyparticle operators for spin operators. For the creation of the multi-particle description, we will use operator representation for spin operators. As an example, we will consider the $X$ component of the spin operator for system of two particles. We will enter designations for states with back as " 0 " state down, and a state with back as a state " 1 " up.

$$
|11>=| \uparrow \uparrow>\text { and }|00>=| \downarrow \downarrow>
$$

Let the operator I acts only the second particle. Then, the action of the operator can be presented in the form:

$$
\begin{aligned}
& 2 \mathrm{I}_{\mathrm{x}}(2)|00>=| 01> \\
& 22 I_{x}(2)|01>=| 00> \\
& 2 I_{x}(2)|10>=| 11> \\
& 2 I_{x}(2)|11>=| 10>
\end{aligned}
$$


If to enter new designations for states $|00\rangle=|0\rangle,|01\rangle=|1\rangle,|10\rangle=|2\rangle,|11\rangle=|3\rangle$, then we will gain the following impression:

$$
\begin{aligned}
& 2 I_{x}(2)|0>=| 1> \\
& 2 I_{x}(2)|1>=| 0> \\
& 2 I_{x}(2)|2>=| 3> \\
& 2 I_{x}(2)|3>=| 2>
\end{aligned}
$$

respectively, in matrix representation, we will receive the operator for the $\mathrm{X}$ components:

$$
2 I_{x}=\left(\begin{array}{llll}
0 & 1 & & \\
1 & 0 & & \\
& & 0 & 1 \\
& & 1 & 0
\end{array}\right)
$$

Repeating these reasonings for other components, we will gain a multi-particle expression for spin operators in a matrix form:

$$
2 I_{y}=\left(\begin{array}{cccc}
0 & i & & \\
-i & 0 & & \\
& & 0 & i \\
& & -i & 0
\end{array}\right) \quad 2 I_{z}=\left(\begin{array}{cccc}
1 & 0 & & \\
0 & -1 & & \\
& & 1 & 0 \\
& & 0 & -1
\end{array}\right)
$$

Further, it is easy to construct similar operators for system of $\mathrm{N}$ particles in the form of $\mathrm{N} \times \mathrm{N}$ matrix.

\subsection{Control of two-particle quantum system}

Below we study the two-particle system - system from two particles with spins. The Hamiltonian for the movement of spin in a magnetic field has a standard form:

$$
H=-\gamma \hbar B I_{z}-\gamma h\left(B^{+} I^{-}+B^{-} I^{+}\right)
$$

where components of a magnetic field are equal 


$$
B^{ \pm}=h_{x} \pm i h_{y}
$$

Here, the raising (lowering) operators have an appearance:

$$
I^{-}=|1><0| \quad I^{+}=|0><1| .
$$

In a matrix form, they have following matrixes $4 \times 4$ :

$$
2 I_{-}=\left(\begin{array}{llll}
0 & 0 & & \\
1 & 0 & & \\
& & 0 & 0 \\
& & 1 & 0
\end{array}\right) \quad 2 I_{+}=\left(\begin{array}{llll}
0 & 1 & & \\
0 & 0 & & \\
& & 0 & 1 \\
& & 0 & 0
\end{array}\right)
$$

We find the solution of Schrödinger's equation in the form as:

$$
\psi(t)=c_{0}(t)\left|0>+c_{1}(t)\right| 1>+c_{2}(t)\left|2>+c_{3}(t)\right| 3>.
$$

Here, variable coefficients are equal:

$$
\begin{gathered}
c_{2}(t)=c_{2}(0) e^{i \omega_{0} t / 2} ; \quad c_{0}(t)=c_{0}(0) e^{i \omega_{0} t / 2} \\
c_{3}(t)=c_{3}(0) e^{i \omega_{0} t / 2} \quad c_{1}(t)=c_{1}(0) e^{-i \omega_{0} t / 2} .
\end{gathered}
$$

And after that we will consider change of population of levels depending on initial conditions. Let us choose the following initial data:

A)

$$
c_{0}(0)=\frac{1}{\sqrt{2}}, \quad c_{1}(0)=\frac{i}{\sqrt{2}} .
$$

The relevant solutions have the following form:

$$
c_{0}(t)=\frac{[\cos (\Omega t / 2)-\sin (\Omega t / 2)]}{\sqrt{2}}, \quad c_{1}(t)=i \frac{[\cos (\Omega t / 2)+\sin (\Omega t / 2)]}{\sqrt{2}}
$$


The probability of staying at the given level is equal:

$$
\left|c_{0}\left(t_{1}\right)\right|^{2}=\frac{1}{2}(1-\sin (\Omega t)), \quad\left|c_{1}\left(t_{1}\right)\right|^{2}=\frac{1}{2}(1+\sin (\Omega t)) .
$$

It is easy to see that also depends on duration of the applied impulse:

$$
\begin{aligned}
& \text { At } t_{1}=\pi / \Omega=2 \pi / \Omega \quad\left|c_{0}\left(t_{1}\right)\right|^{2}=\left|c_{1}\left(t_{1}\right)\right|^{2}=\frac{1}{2} \\
& \text { At } t_{1}=\pi / 2 \Omega \quad\left|c_{0}\left(t_{1}\right)\right|^{2}=0, \quad\left|c_{1}\left(t_{1}\right)\right|^{2}=1 \\
& \text { And at } t_{1}=3 \pi / 2 \Omega \quad\left|c_{0}\left(t_{1}\right)\right|^{2}=1, \quad\left|c_{1}\left(t_{1}\right)\right|^{2}=0
\end{aligned}
$$

According to the obtained results, such a studied configuration with the probability of staying and transition between levels is defined by duration of impulses of a field.

B) Let us use another initial data:

$$
c_{0}(0)=c_{1}(0)= \pm \frac{1}{\sqrt{2}}
$$

So the corresponding solutions have a form:

$$
c_{0}(t)=\frac{\exp (i \Omega t / 2)}{\sqrt{2}}, \quad c_{1}(t)=i \frac{\exp (i \Omega t / 2)}{\sqrt{2}}
$$

The probability of stay at the level is constant and does not depend on impulse duration.

$$
\left|c_{0}\left(t_{1}\right)\right|^{2}=\left|c_{1}\left(t_{1}\right)\right|^{2}=\frac{1}{2} \text {. }
$$

These configurations we will call as absolutely steady. Similar solutions are obtained too under the following initial conditions:

$$
c_{0}(0)=c_{1}(0)= \pm \frac{i}{\sqrt{2}} .
$$


As a result, we have following solutions

$$
c_{1}(t)= \pm \frac{\exp (i \Omega t / 2)}{\sqrt{2}}, \quad c_{0}(t)= \pm i \frac{\exp (i \Omega t / 2)}{\sqrt{2}} .
$$

The probability of stay on these levels is constant and does not depend on impulse duration:

$$
\left|c_{0}\left(t_{1}\right)\right|^{2}=\left|c_{1}\left(t_{1}\right)\right|^{2}=\frac{1}{2}
$$

Thus, in the above part of the chapter, the multi-particle approach to the description of spin system and control of coherent spin states is developed. This multi-particle approach is used for strict description of transfer processes in the quantum systems with many particles. As application of the developed method, the system of two particles with spins is studied. The obvious expressions for spin operators in a multi-particle case are constructed. And control of multi-particle system by the variation of magnetic field is investigated. It is shown that at action by an impulse of a magnetic field and depending on initial data two types of spin states are possible: Stability of first type of states depends on the action of external magnetic field, and second type of spin states is absolutely steady. The second type of states is important for storage and further keeping of the quantum information.

\section{The mathematical formulation of control problem in the system in a magnetic field}

The second part of chapter devoted to more mathematical description of control problem. In the first paragraph, we study the particle (spin) motion in the electromagnetic field; the general equations have obtained. The criteria for optimal time, reaching the desired point, have analyzed, and the calculations for the specific types of control from impulse of the field to the power and Gauss driving force spectrums have made in the two paragraphs. The obtained results shortly discussed in the three paragraphs.

\subsection{The equations of motion within a magnetic field with control}

A particle motion within a magnetic field is known to be described by the following equation:

$$
m \frac{d V}{d t}=\frac{q}{c}[V \times B]+U(t) \frac{q}{c}[V \times h]
$$

where $V$ is the velocity of the particle, $B$ is the constant magnetic field, $m$ is the mass of the particle, $q$ is the charge of the particle, $H(t)=U(t) h$ is the alternating magnetic field, $h$ is the unity vector, and $U(t)$ is the absolute value of the control. 
Considering the equation in components and introducing state vector function $X=\left(x_{1}, x_{2}\right)$, $X \in R^{2}, U \in R$, we may formulate the following model of the controlled system:

$$
\dot{X}=(A+U(t) C) X
$$

where the matrices $A$ and $C$ are defined by the following expressions: $A=\left(\begin{array}{cc}0 & \Omega \\ -\Omega & 0\end{array}\right)$, $C=\left(\begin{array}{cc}0 & \omega \\ -\omega & 0\end{array}\right)$, and parameters $\Omega$ and $\omega-$ by the following ones: $\Omega=\frac{q B}{m c}$ and $\omega=\frac{q h}{m c}$.

As mentioned above, this model describes various physical problems, in particular a particle motion in a magnetic field and spin precession in a magnetic field. From the standpoint of control theory, the task formulated belongs to the degenerate class of the optimal control tasks, since there are additional invariants - the integrals of motion in accordance with the law of conservation of energy:

$$
X_{1}^{2}+X_{2}^{2}=\text { const }, \quad(m=\text { const })
$$

The problem of particle motion control in a magnetic field has formulated as the following: to define the time to reach a given point depending on the parameters of control-the amplitude and the period of control pulse, as well as the specter of external impact. The functional describing the reaching of the given point in space is defined to solve this problem:

$$
L\left(T_{f}\right)=1-\left(\left(x_{1}\left(T_{f}\right), x_{1}^{f}\right)+\left(x_{2}\left(T_{f}\right), x_{2}^{f}\right)\right)
$$

Here, $X^{f}=\left(x_{1}^{f}, x_{2}^{f}\right)$ is final state vector function, $X\left(T_{f}\right)=\left(x_{1}, x_{2}\right)$ is current state vector function. The optimal time to reach the given point has defined by fulfilling the condition of functional extreme (minimum).

The motion of particles within a magnetic field under various external impacts has been considered. The trunk solutions and the dependence of particles phases on the amplitude, duration, and the specter of the impact of fields have been established.

Let us consider the circular particle motion. The simplest way to solve the task is using the complex form. Thus, a complex variable $z=x_{1}+i x_{2}=|z| e^{-i \varphi}$ is introduced, and then the system of the motion equations is transformed to the following simple view:

$$
\frac{d|z|}{d t}=0
$$




$$
\frac{d \varphi}{d t}=\Omega+U(t)
$$

The first equation corresponds to the law of energy conservation (the motion amplitude conservation). The second equation describes the phase alternation while circular motion under the control impact. Thus, the circular motion with the radius specified is completely defined by its phase values. The alternation of the phase is defined by the external impact in turn.

According to Eq. (22), the general solution has such form:

$$
\varphi(t)=\Omega t+\omega \int U(t) d t
$$

\subsection{Control of particle motion}

According to the conditions formulated for optimal control earlier to define the time for reaching the given point, the functional (21) needs to be built.

Consider some point on the circle as the current one.

$$
z=x_{1}+i x_{2}=|z| e^{-i \varphi}
$$

where the phase is defined by the Eq. (22). The final point is chosen to be on the circle for example as at $45^{\circ}$ :

$$
z^{*}=x_{1}^{*}+i x_{2}^{*}=|z| e^{-i \pi / 4}
$$

Then, the functional to be found takes this form:

$$
L\left(T_{f}\right)=1-\frac{y^{2}}{2}\left(1+\sin \left\{2\left(\omega t+\int U(t) d t\right)\right\}\right)
$$

Accordingly, the condition of the functional minimum is defined by the condition for phase:

$$
\varphi\left(T_{F}\right)=\omega T_{F}+\int_{0}^{T_{F}} U(t) d t=2 \pi
$$

Thus, the time for optimal reaching the given point at the $T^{f}$ trajectory is defined by the functional Eq. (24). 


\subsection{The dependence of particles phase on the control amplitude, duration, and specter}

In the absence of external impact, $U(t)$ regular standard solution with the initial angular velocity $\Omega$ arises:

$$
\left\{\begin{array}{l}
V_{x}(t)=V_{0} \cos (\Omega t+\varphi) \\
V_{y}(t)=V_{0} \sin (\Omega t+\varphi)
\end{array},\right.
$$

Then, the time for reaching is defined by the statement:

$$
T_{F}=\frac{\Delta \varphi}{\omega}=\frac{\pi}{4 \omega}
$$

These solutions are known as Trunk solutions [11] in mathematics.

The dependence of the phase of particle circular motion on the control type and duration is analyzed, and the time for reaching is calculated below. Particle motion under various types of external control is considered-see [12] too.

(1) In case of pulse external impact the control function takes the form:

$$
\begin{gathered}
U(t)=U_{0} \theta(t-\varepsilon)-\theta(t) \quad \text { or } \\
U(t)=U_{0} \sum_{n}(t-n \tau)-\theta(t) .
\end{gathered}
$$

Then time $T_{F}$ may be defined from this expression:

$$
\begin{gathered}
2 \pi=\omega T_{F}+u_{0} \tau . \\
T_{F}=\frac{2 \pi-U_{0} \tau}{\omega}=\frac{2 \pi}{\omega}-\tau\left(\frac{\omega_{0}}{\omega}\right)
\end{gathered}
$$

The time for reaching decreases in comparison with the case of impact absence due to the impact amplitude $\omega_{0}$. When the condition fulfilled:

$$
\omega_{0} \tau=2 \pi
$$

reaching is immediate: 


$$
T_{F}=0
$$

(2) In case of exponentially fading impacts the control function takes this form:

$$
U(t)=U_{0} e^{-\alpha t}
$$

In this case, the time to reach the point may be defined by the following:

$$
2 \pi=\omega_{0} T_{F}+\frac{U_{0}}{2}\left[1-e^{(-2 T)}\right]
$$

Consider the cases:

$$
\text { a) } \alpha T_{F}<<1, \text { then } T_{F}=\frac{\pi / 4}{\omega_{0}+\alpha}
$$

In the case of adiabatic impact, the time to reach the point increases.

In case of rapid impact.

$$
\text { b) } \alpha T_{F}>>1 \text {, then } T_{F}=\frac{\pi / 4-\frac{U_{0}}{\alpha}}{\omega}
$$

In the case of Gaussian impact, the control function takes this form:

$$
\begin{array}{r}
U(t)=U_{0} e^{\left(-\beta^{2} t^{2}\right)} . \\
\int_{0}^{T_{F}} U(t) d t=\sqrt{\frac{\pi}{\beta}} \operatorname{erfc}\left(\beta T_{F}\right)
\end{array}
$$

where $\operatorname{erfc}(x)=\int_{0}^{x} e^{\left(-x^{2}\right)} d x$

Consider the following cases: 
a) $\beta T_{F}<<1$, then $(\omega+\beta) T_{F}=\frac{\pi}{4}$, which yields

$$
T_{F}=\frac{\pi}{4(\omega+\beta)}
$$

б) $\beta T_{F}>>1$, then $\omega T_{F}+\frac{U_{0} \sqrt{\pi}}{\beta}=\frac{\pi}{4}$ and

$$
T_{F}=\frac{\frac{\pi}{4}-\frac{U_{0}}{\beta} \sqrt{\pi}}{\omega}
$$

(3) In case of power distribution, the motion function takes this form:

$$
\begin{gathered}
U(t)=U_{0}\left(\frac{t}{\tau}\right)^{\gamma}, \quad \gamma>0 . \\
\int_{0}^{T_{F}} U(t) d t=\frac{U_{0}}{\gamma+1}\left(\frac{t}{\tau}\right)^{\gamma+1}=\frac{1}{\gamma+1} \frac{U_{0}}{\tau^{\gamma}} T_{F}^{\gamma+1} \\
\frac{\pi}{4}=\frac{U_{0}}{(\gamma+1) \tau^{\gamma}} T^{\gamma+1}+\omega T_{F}, \\
\left(\omega_{0} \tau\right)\left(\frac{T_{F}}{\tau}\right)^{1-|\gamma|}+\omega T_{F}=\frac{\pi}{4}, \quad-\infty<\gamma \leq-1 .
\end{gathered}
$$

Consider three cases:

a) $-\infty<\gamma<-1$, then:

$$
\begin{aligned}
\omega_{0} \tau\left(\frac{T_{F}}{\tau}\right)^{1-|\gamma|}+\omega T_{F} & =\frac{\pi}{4} \\
-\omega_{0} \tau\left(\frac{T_{F}}{\tau}\right)^{1-|\gamma|}< & <\omega T_{F},
\end{aligned}
$$

then

$$
T_{F}=\frac{\pi}{4 \omega}
$$

in case of strong impacts. 
In the opposite case of weak impacts

$$
-\omega_{0} \tau\left(\frac{T_{F}}{\tau}\right)^{1-|\gamma|} \gg \omega T_{F},
$$

then

$$
\begin{gathered}
\frac{T_{F}}{\tau}=\left(\frac{\pi}{4 \omega_{0} \tau}\right)^{\frac{1}{|\gamma|-1}} \\
T_{F} \rightarrow \infty .
\end{gathered}
$$

The dependence increases for short time to reach the point.

б) $-1<\gamma<0$, than:

$$
\left(\omega_{0} \tau\right)\left(\frac{T_{F}}{\tau}\right)^{\gamma+1}+\omega T_{F}=\frac{\pi}{4}
$$

At short times, time to reach the point does not depend on the specter of time distribution:

$$
\left(\omega_{0} \tau\right)\left(\frac{T_{F}}{\tau}\right)^{1-|\gamma|}+\omega T_{F}=\frac{\pi}{4}
$$

When $T_{F} \rightarrow \infty$

$$
T_{F}=\left(\frac{\pi}{4} \frac{1}{\omega_{0} \tau}\right)^{\frac{1}{1-|\gamma|}}
$$

At large times, time to reach the point is defined by the specter of distribution.

c) $\gamma>0$

then $\left(T_{F}\right)^{\gamma+1}+\omega T_{F}=\frac{\pi}{4}$, that yields

$$
T_{F}=\tau\left(\frac{\pi}{4 \omega_{0} \tau}\right)^{\frac{1}{\gamma+1}}
$$


At short times with low impact, time to reach the point is not sensitive to the specter and then $T_{F}=\frac{\pi}{4 \omega}$.

At large times with strong impact, time to reach the point is defined by the type of the specter.

\section{Conclusion}

Thus, in this chapter, the multi-particle approach to the description of spin system and control of coherent spin states is developed. Obvious expressions for spin operators in a multi-particle case are constructed. On the example of two-partial system, control of system by the variation of magnetic field is investigated. It has shown that at action by an impulse of a magnetic field and depending on initial data two types of spin states are possible: conditionally steady and absolutely steady. The second type of states is important for storage of quantum information.

Thus, the second part of the chapter describes particle motion both without and under the impact of external fields. The possibility to control the motion and reaching of the given point under different types of external impact is investigated. The dependence of time to reach the given point at the trajectory on the specter of the impact has been established; this dependence has particularly been expressed for the power specter also.

\section{Acknowledgements}

Work is performed with partial financial support Russian Ministry of Education and Science (Grant No. 3484)

\section{Author details}

Valeriy Efimovich Arkhincheev ${ }^{1,2}$

Address all correspondence to: varkhin@mail.ru

1 Buryat State University, Ulan-Ude, Russian Federation

2 State Scientific Institution Buryat Research Institute of Agriculture, Ulan-Ude, Russia

\section{References}

[1] Valiev K.A. Quantum computers and quantum calculations. Advances in Physical Sciences. Uspekhi fizicheskih nauk (in Russian) 2005;175:3-39. 
[2] Shor P.W. Algorithms for Computation: Discreta Logarithms and Factoring. Proceedings of the 35th Annual Symposium on the Foundation of Computer Science. Los Alamitos, CA, USA, 1994. pp. 124-134.

[3] Shor P.W., Preskil J. Simple proof of security of the BB84 quantum key distribution protocol, 2000, http://www.arxiv.org/abs/quant-ph/0003004 .

[4] Shor P.W., Smolin J.A. Quantum error-correcting codes need not completely reveal the error syndrome, 1996, http://arxiv.org/abs/quant-ph/9604006.

[5] Nilsen M.A., Chuang I.L. Quantum calculations and quantum information. Cambridge: Cambridge University Press, 2000.

[6] Ekert A., Jozsa R. Quantum computation and Shor's factoring algorithm. Rev Mod Phys. 1996;68:1.

[7] Gruska J. Quantum computing. London: McGraw Hill, 1999.

[8] Imre S., Balazs F. Quantum computing and communications. Chichester: John Willey \& Sons. Ltd., 2005.

[9] Feynman R.P. Simulating physics with computers. Int J Theor Phys. 1982; 21:467.

[10] Grover L.K. A fast quantum mechanical algorithm for database search. Proceedings of the 28th Annual ACM Symposium on Theory of Computation (Philadelphia, Pennsylvania). New York: ACM Press, 1996. pp. 212-218.

[11] Gurman V. Transformations of controlled systems for studying impulse regimes. Avtomatika i Telemekhanika. Autom Telemeh (in Russian). 2009;4:89-97.

[12] Arkhincheev V.E. Chemical physics and mesocopics (in Russian), Chemical physics and mesocopics 2013;15(1):101-104. 